This paper is a preprint (IEEE "accepted" status). IEEE copyright notice. (C) 2020 IEEE. Personal use of this material is permitted. Permission from IEEE must be obtained for all other uses, in any current or future media, including reprinting/republishing this material for advertising or promotional purposes, creating new collective works, for resale or redistribution to servers or lists, or reuse of any copyrighted.

To cite this paper please refer to

A. Giuseppi, A. Pietrabissa, "Wardrop Equilibrium in Discrete-Time Selfish Routing with TimeVarying Bounded Delays", IEEE Transactions on Automatic Control (IEEE), 2020, pp. 1-12, DOI: 10.1109/TAC.2020.2981906 


\title{
Wardrop Equilibrium in Discrete-Time Selfish Routing with Time-Varying Bounded Delays
}

\author{
Alessandro Giuseppi, Antonio Pietrabissa
}

\begin{abstract}
This paper presents a multi-commodity, discretetime, distributed and non-cooperative routing algorithm, which is proved to converge to an equilibrium in the presence of heterogeneous, unknown, time-varying but bounded delays. Under mild assumptions on the latency functions which describe the cost associated to the network paths, two algorithms are proposed: the former assumes that each commodity relies only on measurements of the latencies associated to its own paths; the latter assumes that each commodity has (at least indirectly) access to the measures of the latencies of all the network paths. Both algorithms are proven to drive the system state to an invariant set which approximates and contains the Wardrop equilibrium, defined as a network state in which no traffic flow over the network paths can improve its routing unilaterally, with the latter achieving a better reconstruction of the Wardrop equilibrium. Numerical simulations show the effectiveness of the proposed approach.
\end{abstract}

Index Terms - Wardrop equilibrium, LaSalle's invariance principle, selfish routing, time-delay systems

\section{INTRODUCTION}

$\mathrm{W}$ ARDROP equilibria are a game-theoretical concept, originally introduced for network games when modelling transportation networks with congestion [1]. A system is said to have attained a Wardrop equilibrium, in its standard adversarial formulation, when "the journey times on all the routes actually used are equal, and less than those which would be experienced by a single vehicle on any unused route" [2]. In dynamic selfish routing algorithms, each agent (e.g., cars in transportation networks, packets in communication ones) makes its decision for its own interest, i.e., without taking into account the congestion and the consequent performance degradation it causes to the other agents with its decisions [3].

The dynamic, discrete-time, algorithm presented in this paper deals with a multi-commodity flow problem that consists of distributing a flow demand, split between various source and destination facilities, over a communication network. The convergence of the controlled network state to a set that approximates the Wardrop equilibrium is proven by means of standard control theory arguments, derived from LaSalle's

This work was supported in part by the European Commission in the framework of the H2020 EU-Korea project 5G-ALLSTAR (5G AgiLe and fLexible integration of SaTellite And cellular, www.5g-allstar.eu/) under Grant Agreement no. 815323. invariance principle.

The network is modelled as a time-invariant communication graph and the total flow demand is constituted by various constant traffic flows, or commodities, each one characterised by a source and a destination node. The selfish routing problem is then solved by distributing the flow demand over the admissible network paths so that each commodity unilaterally decides its routing. The congestion state of an edge of the network is characterised by a cost function, referred to as edge latency, that captures the flow distribution performances.

In the scenario described so far, the various commodities are constituted by an infinite stream of infinitely-many arriving agents, each being responsible for an infinitesimal amount of traffic, or job, and each one being able to make an individual decision regarding its routing over the available paths that connect its source to its destination. Within the formulated selfish non-cooperative routing problem, the sought equilibrium is a generalization of the Nash equilibrium, in which no agent can improve its decision unilaterally, to the case in which infinitely many agents compete [4], i.e., to a scenario in which the individual decision of a single agent has no significant impact on the performances of the others.

Firstly, it is assumed that each agent is not provided with a model of the network and has to make its routing decisions based only on the measures of the latencies associated to the network edges traversed by the paths of the commodity it belongs to. Furthermore, it is assumed that the latency measures are subject to time-varying, unknown but bounded delays. Under such assumptions, the proposed routing algorithm is proved to converge to an approximation of the Wardrop equilibrium. This paper further discusses the case in which the agents have information also on the latencies of the paths used by the other commodities, proving that, in this case, the system is driven to a better approximation of the Wardrop equilibrium.

The rest of the paper is organised as follows: Section II presents the state of the art on selfish routing solutions and their relation with Wardrop equilibria, and highlights the contributions of this work; Section III contains the selfish routing problem formulation; Section IV presents the proposed discrete-time control law and discusses some useful lemmata; Section $\mathrm{V}$ reports the convergence analysis of the proposed

A. Giuseppi and A. Pietrabissa are joint first authors and are with the Department of Computer, Control, and Management Engineering Antonio Ruberti, University of Rome La Sapienza, via Ariosto 25, 00162, Rome, Italy (email: \{giuseppi, pietrabissa\}@diag.uniroma1.it). 
control solution and proves the convergence of the network to a set of approximated Wardrop equilibria; Section VI proposes an algorithm improvement in presence of (limited) information exchanges; Section VII validates the approach by discussing the results of numerical simulations, while Section VIII draws the conclusions and discusses possible future research directions.

\section{RELATED WORK AND PROPOSED INNOVATION}

Distributing flow is a fundamental aspect of any network control and operation problem and can be particularised in order to address several domains, spacing from communication systems [5] to power [6] and traffic [7], [8] networks. The problem of selfish routing arises in networks in which the flow is constituted by autonomous agents [9], as no central entity is responsible for the flow distribution nor can directly influence it without modifying the network itself, for example by introducing routing tolls or flow capacity limitations [10]. In such scenarios, the network flow distribution and network congestion are entirely dependent on the decisions of the various agents and their objectives.

In the framework of selfish routing algorithms, a significant role is played by the so-called Wardrop equilibrium [1], which represents a network state in which no agent can unilaterally improve its objective. In Wardrop's typical routing problem formulation [11], the traffic model envisages that the travel time, or latency, associated with the network paths is a nondecreasing function of their corresponding flow, and the Wardrop equilibrium is consequently reached when all used roads present an equalised travelling time. With adequate considerations, the original definition of Wardrop equilibrium can be extended also to capacitated networks [12], [13], uncertain networks [14], [15] and time-varying ones [16].

It is well known that Wardrop equilibria can be computed by centralised algorithms in polynomial time [17], but, due to the inherently distributed nature of the network flow distribution, a significant research effort was spent developing distributed and dynamic solutions to drive the network to a Wardrop equilibrium, as in [11], [16], [18]-[25]. This paper, as most of the aforementioned ones, considers a dynamic communication network [22], [23] whose evolution is governed by a set of differential equations derived by mass/flow conservation laws.

Several of the works available in the literature utilise the concepts of learning and exploration, as it is common to assume limited knowledge regarding the state and the characteristics of the network. In general, these approaches are built around the idea of sampling different flow distribution strategies to increase the knowledge of the environment, and then exploit it to converge to certain desired states. For instance, in [18] an asynchronous and distributed solution is presented, in which transmission probabilities are updated following a reinforcement learning approach, depending on an estimation of the network edges latencies. The authors of [11] prove, by Lyapunov arguments, the convergence of an iterative distributed learning solution to a Wardrop equilibrium state.

Assuming an exchange of information regarding the congestion state of the network among agents, Fischer et al. present in [19] a round-based algorithm, in which a finite number of players, each one responsible for a different commodity, redistribute the traffic flow following a policy that assures the convergence to an approximated equilibrium of the same nature of the ones considered in this work. A similar information exchange, based on an ideal bulletin board, is proposed by the same authors also in [20], where an approximate Wardrop equilibrium is reached, under assumptions similar to the ones of this paper, even if the agents are presented with stale, or delayed, information regarding the network state. In [21], a round-based formulation of the algorithm in [20] is proposed.

The present work extends the results of previous works from the authors [24], [25] and the ones available in literature mainly in two directions: (i) the asymptotic analysis and algorithm design are extended to the multi-commodity case, which is not explicitly discussed in the cited works, enabling the application to more realistic scenarios; (ii) the agents of the various commodities are provided with a measure of the network state subject to heterogeneous, time-varying, unknown but bounded delays; in this respect, differently from [20], which only analyses the tolerance to delays of the algorithm developed therein, here the delays are explicitly considered and compensated within the algorithm development.

\section{Multi-COMmodity SElfish Routing Problem}

Standard notation is used throughout the paper, with $|\cdot|$ denoting the cardinality operator.

\section{A. Preliminaries on Wardrop routing}

The modelling framework utilised in this paper is derived from the one in [21], commonly used in selfish-routing problems considering an infinite population of agents, each one carrying an infinitesimal amount of flow. In practice, in the case for example transportation or communication networks, a single vehicle or packet, respectively, is approximately considered as an agent: in fact, even if the number of vehicles/packets is finite, if the flow rates are sufficiently high, the population approximates the infinite population assumed by Wardrop theory [1].

We are given a network $\mathcal{G}=(\mathcal{V}, \mathcal{E})$, where $\mathcal{V}$ is the finite set of vertices or nodes, $\mathcal{E} \subseteq \mathcal{V} \times \mathcal{V}$ is the set of edges or links. Let $\mathcal{C}$ denote a set of commodities with constant traffic demands $d^{i}>0, \forall i \in \mathcal{C}$, generally expressed in jobs per unit of time, with total demand $d:=\sum_{i \in \mathcal{C}} d^{i}$. For each commodity, the source node is connected by the network to the destination node through the set of paths $\mathcal{P}^{i}$. Let $\mathcal{P}:=\bigcup_{i \in \mathcal{C}} \mathcal{P}^{i}$ be the set of all the network paths. A given path $p \in \mathcal{P}^{i}$ includes a set of links; let $\mathcal{P}_{e}$ be the set of paths traversing the edge $e$, i.e., $\mathcal{P}_{e}:=$ $\{p \in \mathcal{P} \mid e \in p\}$, let $\mathcal{P}_{e}^{i}:=\mathcal{P}_{e} \cap \mathcal{P}^{i}$ and let $\mathcal{E}^{i}$ be the set of edges traversed by the paths in $\mathcal{P}^{i}$, i.e., $\mathcal{E}^{i}:=\left\{e \in p \mid p \in \mathcal{P}^{i}\right\}$. For each commodity $i \in \mathcal{C}$, let us also define the maximum number of paths traversing an edge as $\eta^{i}:=\max _{e \in \mathcal{E}^{i}}\left|\mathcal{P}_{e}\right|$.

In this modelling framework, an agent is considered to be, as in [11], an infinitesimal portion of a specified commodity. Let $x_{p}^{i}$ be the volume of the agents, or bandwidth, of commodity $i$ 
relying on path $p \in \mathcal{P}^{i}$. The vector $\boldsymbol{x}:=\left(x_{p}^{i}\right)_{p \in \mathcal{P}^{i}, i \in \mathcal{C}}$ is the flow vector or population share, whose components specify the overall amount of traffic per unit of time flowing along path $p \in$ $\mathcal{P}^{i}$ and associated with commodity $i \in \mathcal{C}$. Let $x_{p}:=\sum_{i \in \mathcal{C}} x_{p}^{i}$, $x_{e}^{i}:=\sum_{p \in \mathcal{P}^{i} \cap \mathcal{P}_{e}} x_{p}^{i}$ and $x_{e}:=\sum_{i \in \mathcal{C}} x_{e}^{i}$ denote the total traffic flow over path $p \in \mathcal{P}$, the traffic flow of commodity $i$ over edge $e$ and the total traffic flow over edge $e$, respectively.

Definition 1. The feasible state space, i.e., the compact set of feasible flow vectors, is

$X:=\left\{\boldsymbol{x} \in \mathbb{R}^{|\mathcal{P}| \times|\mathcal{C}|} \mid x_{e} \geq 0, \forall e \in \mathcal{E}\right.$ and $\left.\sum_{p \in \mathcal{P}} x_{p}^{i}=d^{i}, \forall i \in \mathcal{C}\right\}$.

A metric of interest is the average response time required by the path $p \in \mathcal{P}$ for serving an amount of traffic equal to $x_{p}$.The response time grows with the considered traffic flow and thus is a reliable indicator of the path congestion status. Hence, this quantity is defined as the latency function associated with path $p \in \mathcal{P}$ and is a non-negative function $l_{p}(\boldsymbol{x}):[0, d] \rightarrow \mathbb{R}_{\geq 0}$. The path latency is the sum of the latencies of the edges of the path, denoted with $\quad l_{e}\left(x_{e}\right):[0, d] \rightarrow \mathbb{R}_{\geq 0}, \quad$ i.e., $\quad l_{p}(\boldsymbol{x})=$ $\sum_{e \in p} l_{e}\left(x_{e}\right), \forall p \in \mathcal{P}$. The shape of the latency functions depends on the application considered. One strength of the proposed approach is that the agents only rely on measures of the latency functions, which are not required to be explicitly modelled. The latency functions are only assumed to have the following properties.

Assumption 1. The latency functions $l_{e}(\xi), \forall e \in \mathcal{E}$, are Lipschitz continuous and strictly increasing over the interval $[0, d]$. Let $\beta_{e}$ be the local Lipschitz constant of $l_{e}, \beta_{p}:=\operatorname{sum}_{e \in p} \beta_{e}$ and $\bar{\beta}^{i}:=\max _{p \in \mathcal{P}^{i}} \beta_{p}$.

Assumption 1 is a reasonable restriction since the response time of an edge generally increases with the total amount of traffic flow routed onto that edge. Note that the latency of a path $p \in \mathcal{P}$ is a function of the flow vector $\boldsymbol{x}$, and the latency of an edge is a function of the flow $x_{e}$ routed over edge $e$.

The agents' aim is that of minimizing their personal latency selfishly, without considering the impact on the global situation. The routing problem is formulated below as the problem of determining the strategies which will lead the flow vector to reach a Wardrop equilibrium. In Wardrop theory, stable flow assignments are the ones in which no agent can improve its situation by changing its strategy (i.e., the set of used paths) unilaterally. This objective is achieved if the network reaches a Wardrop equilibrium.

Definition 2 ([19]). A feasible flow vector $\boldsymbol{x}$ is at a Wardrop equilibrium if, for each path $p \in \mathcal{P}^{i}$ such that $x_{p}^{i}>0$, the following relation holds: $l_{p}(\boldsymbol{x}) \leq l_{q}(\boldsymbol{x}), \forall q \in \mathcal{P}^{i}, \forall i \in \mathcal{C}$.

\footnotetext{
${ }^{1}$ In case the latency functions are increasing, there are more Wardrop equilibria; a convergence analysis under this milder assumption can be found
}

The set of Wardrop equilibria is then defined as

$$
\begin{gathered}
\mathcal{X}_{\mathcal{W}}:=\left\{\boldsymbol{x} \in \mathcal{X} \mid l_{p}(\boldsymbol{x})-l_{q}(\boldsymbol{x}) \leq 0, \text { for all pairs } p, q \in\right. \\
\left.\mathcal{P}^{i} \text { s.t. } x_{p}^{i}>0, \text { for all } i \in \mathcal{C}\right\} .
\end{gathered}
$$

In practice, at the Wardrop equilibrium, the latencies of all the loaded paths for each commodity $i \in \mathcal{C}$ have the same value; therefore, provided that the latency functions properly represent the path performances, a fair exploitation of the network resources is achieved by driving the flows towards a Wardrop equilibrium.

In the framework of researches on Wardrop equilibria, a key role is played by the Beckmann, McGuire, and Winsten potential [17].

$\Phi(\boldsymbol{x}):=\sum_{e \in \mathcal{E}} \int_{0}^{x_{e}} l_{e}(\xi) d \xi$,

whose properties are summarised by Property 1 below.

Property 1 ([20]) Under Assumption 1, the potential (2) is continuous and the following properties hold:

i) there exists a unique flow, denoted with $\boldsymbol{x}_{e q}$, over the set of feasible flows, minimizing $\Phi$;

ii) correspondingly, there exists a unique, positive, minimum $\Phi_{\min }=\Phi\left(\boldsymbol{x}_{e q}\right)>0$

iii) $\boldsymbol{x}_{e q}$ is at the Wardrop equilibrium.

Property 1 implies that, under Assumption 1, the set $x_{\mathcal{W}}$ collapses into a unique Wardrop equilibrium ${ }^{1}$ with jobs vector $\boldsymbol{x}_{e q}$.

\section{B. Preliminaries on Stability Results}

The system of interest is a discrete-time system described by

$\boldsymbol{x}[k+1]=f(\boldsymbol{x}[k]), k=0,1, \ldots$,

with $\boldsymbol{x}[k] \in \mathcal{X} \subset \mathbb{R}^{n}, \forall k \geq 0$. Under Assumption 1, let $\boldsymbol{x}_{e q} \in$ $x$ be the unique equilibrium state and let us define the Lyapunov function $V(\boldsymbol{x})$ as follows:

Definition 3. $\mathcal{L}: \mathcal{X} \rightarrow \mathbb{R}$ is a candidate Lyapunov function for the discrete-time nonlinear system (3) if

i) $\mathcal{L} \in \mathcal{C}^{1}$ and is bounded below on $\mathcal{X}$,

ii) $\mathcal{L}(\boldsymbol{x})$ is positive definite on $\mathcal{X}$, with $\mathcal{L}\left(\boldsymbol{x}_{e q}\right)=0$ and $\mathcal{L}(\boldsymbol{x})>0$ if $\boldsymbol{x} \neq \boldsymbol{x}_{e q}$

iii) $\mathcal{L}$ satisfies

$$
\Delta \mathcal{L}(\boldsymbol{x}[k]):=\mathcal{L}(f(\boldsymbol{x}[k]))-\mathcal{L}(\boldsymbol{x}[k]) \leq 0, k=0,1,2, \ldots
$$

along forward trajectories of (3).

The LaSalle invariance principle for discrete-time nonlinear

in [24]. In this paper, strictly increasing functions are assumed for the sake of simplicity. 
systems in [26], [27] will be used to prove the algorithm convergence:

Theorem 1 ([26]). Let $\mathcal{L}(\boldsymbol{x})$ be a candidate Lyapunov function for the discrete-time nonlinear system (3). Then, any bounded trajectory tends to the largest invariant subset $\mathcal{M}$ contained in the locus of points defined by $\Delta \mathcal{L}(\boldsymbol{x})=0$.

\section{PROPOSED CONTROLlER AND SOME USEFUL LEMMATA}

This section presents the proposed control law (Section IV.A) and some lemmata (Section IV.B) which will be used for the convergence proof of Section $\mathrm{V}$.

\section{A. Definition of the Control System and of the Proposed} Control law

As further discussed afterwards, the proposed control law aims at achieving an approximate Wardrop equilibrium within the set defined as follows:

Definition 4. The set of $(\varepsilon, \delta)$-Wardrop equilibria is defined as

$\mathcal{X}_{\mathcal{W}}^{\varepsilon, \delta}:=\left\{\boldsymbol{x} \in \mathcal{X} \mid l_{p}(\boldsymbol{x})-l_{q}(\boldsymbol{x}) \leq \varepsilon\right.$, for all pairs $p, q \in$

$\mathcal{P}^{i}$ s.t. $x_{p}^{i}>\delta$, for all $\left.i \in \mathcal{C}\right\}$,

where $\varepsilon \geq 0$ represents a tolerated latency mismatch and $\delta \geq 0$ represents a significance threshold on the path population.

At the $(\varepsilon, \delta)$-Wardrop equilibrium, the values of latencies of all the paths $p \in \mathcal{P}^{i}$ of each commodity $i \in \mathcal{C}$ whose population $x_{p}^{i}$ is larger than $\delta$, hereafter referred to as $\delta$-loaded paths, differ at most by $\varepsilon$. As explained in Section IV.B, the parameter $\delta$ is here introduced to cope with the multi-commodity scenario.

We consider a discrete-time time-delay control system, where, periodically every $\tau$ seconds, the commodities receive the updated measures of the latency values. At time $k$, each commodity $i \in \mathcal{C}$ receives the measure of the latency of path $p$ with a delay $h_{p}^{i}[k]$, expressed as an integer number of sample times, for all $p \in \mathcal{P}^{i}$. The delays are unknown, time-varying and bounded by the known delay upper-bound $\bar{h}$ :

$$
h_{p}^{i}[k] \in \mathcal{H}, \forall p \in \mathcal{P}^{i}, \forall i \in \mathcal{C}, k=0,1, \ldots
$$

with $\mathcal{H}:=\{0,1, \ldots, \bar{h}\}$. The delays are collected in the delay vector $\boldsymbol{h}[k] \in \mathcal{H}^{\mathcal{C} \times \mathcal{P}}$.

Whenever clear from the context, for the sake of notation simplicity, the latency $l_{p}(x[k])$ will be simply denoted as $l_{p}[k]$.

The system dynamics is described by

$x_{p}^{i}[k+1]=x_{p}^{i}[k]+\tau \sum_{q \in \mathcal{P}}\left(r_{q p}^{i}[k]-r_{p q}^{i}[k]\right), k=0,1, \ldots$,

where $r_{p q}[k]$ is the control action and is the so-called migration rate from path $p \in \mathcal{P}^{i}$ to path $q \in \mathcal{P}^{i}$. Similarly to a large part of selfish-routing algorithms in the literature [19], the proposed control law is

$r_{p q}^{i}[k]=x_{p}^{i}[k] \sigma_{p q}^{i}[k] \mu_{p q}^{i}[k]$,

where $\mu_{p q}^{i}[k]$ is the so-called migration policy, representing the decision whether a population share of commodity $i$ assigned to path $p$ migrates to path $q$, and $\sigma_{p q}^{i}[k]$ is interpreted as the (positive) control gain and sets the rate with which the population share of path $p$ migrates to path $q$.

There is a variety of migration policies used in the literature for delay-free continuous-time setups, such as the better response policy

$$
\mu_{p q}^{i}(t)= \begin{cases}0 & \text { if } l_{p}(\boldsymbol{x}(t))-l_{q}(\boldsymbol{x}(t)) \leq 0 \\ 1 & \text { otherwise }\end{cases}
$$

However, by using these standard migration policies in the discrete-time, it can be shown that convergence cannot be guaranteed even in the delay-free case, however small the sampling time $\tau$ is chosen [20]. In [24], a modified better response policy to achieve an $\varepsilon$-Wardrop equilibrium in the discrete-time, single-commodity case (corresponding to a $(\varepsilon, 0)$-Wardrop equilibrium, as per definition (4)) is proposed:

$$
\mu_{p q}^{i}[k]=\left\{\begin{array}{cc}
0 & \text { if } l_{p}[k]-l_{q}[k] \leq \varepsilon \\
\mu_{p q}\left(l_{p}[k], l_{q}[k]\right), & \text { otherwise }
\end{array},\right.
$$

where $\mu_{p q}\left(l_{p}[k], l_{q}[k]\right)$ is a bounded positive real-valued function of the latency measures.

Here, we further modify the migration policy of [24] by considering the latency measures instead of the current latency values to cope with the delays:

$\mu_{p q}^{i}[k]=\left\{\begin{array}{l}0 \text { if } l_{p}\left(\boldsymbol{x}\left[k-h_{p}^{i}[k]\right]\right)-l_{q}\left(\boldsymbol{x}\left[k-h_{q}^{i}[k]\right]\right) \leq \varepsilon \\ 1 \text { otherwise }\end{array}\right.$

where $l_{p}\left(\boldsymbol{x}\left[k-h_{p}^{i}[k]\right]\right)$ is the last measure of $l_{p}$ available to commodity $i$ and with

$0<\varepsilon \leq \min _{i \in \mathcal{C}} \bar{\beta}^{i} d^{i}|\mathcal{C}|$

This bound on $\varepsilon$ has a simple interpretation in the single commodity case, i.e., when $|\mathcal{C}|=1$, since $\bar{\beta}^{i} d^{i}$ represents the maximum latency value that can be achieved by any path $p \in$ $\mathcal{P}^{i}$ when the population of commodity $i$ is totally conveyed over a single path: if $\varepsilon>\bar{\beta}^{i} d^{i}$ any flow distribution is therefore at the $(\delta, \varepsilon)$-equilibrium.

The proposed controller is expressed by equations (6), (7), with the following control gain:

$\sigma_{p q}^{i}[k]=\frac{\sigma}{\tau d^{i} \bar{\beta}^{i}\left(\left|\mathcal{P}^{i}\right|+\eta^{i}\right)|\mathcal{C}|(1+\bar{h})}$,

with 
$\sigma \leq \varepsilon$

The controller design parameters are therefore $\sigma$ and $\varepsilon$.

We consider that, initially, the population of each commodity is distributed over all its network paths, i.e.,

$\left\{\begin{array}{l}x_{p}^{i}[-h]=x_{p}^{i}[0]>0 \\ \sum_{p \in P^{i}} x_{p}^{i}[0]=d^{i}\end{array}, \forall p \in \mathcal{P}^{i}, \forall i \in C, \forall h \in \mathcal{H}\right.$.

Recalling the concepts of exploration/exploitation, the first condition of (11) may be interpreted as the need for the commodity $i$ to explore all the available paths to perform the latency measures ${ }^{2}$.

B. Some Useful Lemmata and Definition of the Augmented State Space

The following Lemma will be useful for the convergence proof presented in Section V.

Lemma 1. Under Assumption 1, considering the dynamics (5) under the control law (6), (7), (9), with parameters set as in (8) and (10), the latency variation of a path $p \in \mathcal{P}$ in one timestep is bounded as

$-\frac{\sigma}{|C|(1+\bar{h})} \sum_{i \in C} \frac{\eta^{i}}{\left|\mathcal{P}^{i}\right|+\eta^{i}} \leq l_{p}(f(\boldsymbol{x}[k]))-l_{p}(\boldsymbol{x}[k])$

$\leq \frac{\sigma}{|C|(1+\bar{h})} \sum_{i \in C} \frac{\left|\mathcal{P}^{i}\right|}{\left|\mathcal{P}^{i}\right|+\eta^{i}}$.

\section{Proof (see Appendix 1).}

A straightforward consequence of equation (12) of Lemma 1 is that the decrease in one step of the difference between the latency values of two paths $p, q \in \mathcal{P}$ is limited from below by

$\left(l_{p}[k+1]-l_{q}[k+1]\right)-\left(l_{p}[k]-l_{q}[k]\right)$

$\geq-\frac{\sigma}{|\mathcal{C}|(1+\bar{h})} \sum_{i \in \mathcal{C}}\left(\frac{\eta^{i}}{\left|\mathcal{P}^{i}\right|+\eta^{i}}+\frac{\left|\mathcal{P}^{i}\right|}{\left|\mathcal{P}^{i}\right|+\eta^{i}}\right)=-\frac{\sigma}{1+\bar{h}}$.

Before proving the next lemma, we define an augmented state to account for the bounded delays:

$\boldsymbol{z}[k]=\left(\boldsymbol{z}_{0}[k] \boldsymbol{z}_{1}[k] \ldots \boldsymbol{z}_{\bar{h}}[k]\right)^{T}$

$=(\boldsymbol{x}[k] \boldsymbol{x}[k-1] \ldots \boldsymbol{x}[k-\bar{h}])^{T}$,

with

$\boldsymbol{z}_{h}[k]=\left(z_{p h}[k]\right)_{p \in \mathcal{P}}=\left(x_{p}[k-h]\right)_{p \in \mathcal{P}}=\boldsymbol{x}[k-h]$,

$z_{p h}[k]=\sum_{i \in \mathcal{C}} z_{p h}^{i}[k], z_{p h}^{i}[k]:=x_{p}^{i}[k-h]$.

The augmented state space is defined as

\footnotetext{
${ }^{2}$ If each commodity $i \in \mathcal{C}$ receives the latency measures of all the paths $p \in$ $\mathcal{P}^{i}$ even if $x_{p}^{i}[k]=0$, it is sufficient that the initial conditions are feasible, i.e.,
}

$Z:=\left\{\mathbf{z}=\left(\mathbf{z}_{h}\right)_{h \in \mathcal{H}} \mid \mathbf{z}_{h} \in \mathcal{X}, \forall h \in \mathcal{H}\right\}=\mathcal{X}^{\bar{h}}$,

the unique Wardrop equilibrium as

$\boldsymbol{z}_{\mathcal{W}}=\left(\mathbf{z}_{h}\right)_{h \in \mathcal{H}}$, with $\mathbf{z}_{h}=\boldsymbol{x}_{\mathfrak{W}}, \forall h \in \mathcal{H}$

and the system dynamics as

$$
\left\{\begin{array}{rl}
z_{p 0}[k+1] & =z_{p 0}[k]+\tau \sum_{q \in P}\left(r_{p q}[k]-r_{q p}[k]\right) \\
z_{p 1}[k+1] & =z_{p 1}[k] \\
& \cdots \\
z_{p \bar{h}}[k+1] & =z_{p(\bar{h}-1)}[k]
\end{array}, \forall p \in P\right.
$$

with initial conditions (from equation (11))

$\boldsymbol{z}[0]=(\boldsymbol{x}[k])_{k=-\bar{h},-\bar{h}+1, \ldots, 0} \in \mathcal{Z}$.

The augmented system dynamics (18), (19), under the control law (6)-(10), will be hereinafter referred to as augmented selfish routing (ASR) dynamics. The ASR dynamics yields, in vector form, the closed-loop nonlinear system dynamics

$\boldsymbol{z}[k+1]=g(\boldsymbol{z}[k])=\left(\begin{array}{c}f\left(\mathbf{z}_{0}[k]\right) \\ \boldsymbol{z}_{0}[k] \\ \ldots \\ \mathbf{z}_{(\bar{h}-1)}[k]\end{array}\right)$.

Such a map from a population game to a set of difference equations is referred to in the literature as deterministic evolutionary dynamics [28].

The stability analysis reported in Section V is conducted with respect to the ASR dynamics. Preliminarily, we show that the state space $Z$ is a positively invariant set.

Lemma 2. $Z$ is a positively invariant set for the ASR dynamics.

\section{Proof (see Appendix 1).}

Let us define the function

$\mathcal{L}(\mathbf{z}):=\sum_{h \in \mathcal{H}} L\left(\mathbf{z}_{h}\right)$, with $L(\boldsymbol{x}):=\Phi(\boldsymbol{x})-\Phi_{\text {min }}$,

where $\Phi(\boldsymbol{x})$ is the potential (2) and $\Phi_{\min }$ is its minimum value, which is unique, under Assumption 1, thanks to Property 1. Let also

$\Delta \mathcal{L}(\boldsymbol{z}[k])=\sum_{h \in \mathcal{H}} \Delta L(\boldsymbol{x}[k-h])=\sum_{h \in \mathcal{H}} \Delta L\left(\boldsymbol{z}_{h}[k]\right)=$

$=\sum_{h \in \mathcal{H}}\left(\Phi\left(f\left(\mathbf{z}_{h}[k]\right)\right)-\Phi\left(\mathbf{z}_{h}[k]\right)\right), k=0,1, \ldots$

denote the difference of the function $\mathcal{L}(\boldsymbol{z}[k])$ along the

equations (11) can be substituted by $\boldsymbol{x}[-h] \in \mathcal{X}, \forall h \in \mathcal{H}$. 
solutions of the ASR system.

Lemma 3. Under Assumption 1, function (21) is a candidate Lyapunov function (as in Definition 3) for the ASR system.

\section{Proof (see Appendix 1).}

\section{CONVERGENCE RESUlT}

The main convergence result is expressed by Theorem 3 .

Theorem 3. Under Assumptions 1, the ASR dynamics robustly asymptotically converges to an $(\varepsilon, \delta)$-Wardrop equilibrium under heterogeneous, time-varying, unknown but bounded delays $\boldsymbol{h}[k] \in \mathcal{H}^{\mathcal{C} \times \mathcal{P}}$.

\section{Proof (see Appendix 1).}

Recalling that $z_{0} \in \mathcal{X}_{\mathcal{W}}^{(\varepsilon, \delta)}$ for all $\mathbf{z} \in \mathcal{M}$, Theorem 1 entails that $\boldsymbol{x}[k]=\boldsymbol{z}_{\mathbf{0}}[k]$ tends to the set $\chi_{\mathcal{W}}^{(\varepsilon, \delta)}$, i.e., to an $(\varepsilon, \delta)-$ Wardrop equilibrium.

\section{CONTROL LAW WITH COMMUNICATIONS}

From the discussions of the previous section, it is straightforward to think of reducing the latency tolerance with $k$, i.e., to have a time-varying $\varepsilon[k]$ in (7), in order to force the system to converge to a shrinking set $\mathcal{X}_{\mathcal{W}}^{\varepsilon[k], \delta}$. The advantage would be twofold: initially, when the latency differences are large, one could use a large value for $\varepsilon[k]$ improving the convergence velocity (we recall that the control gain (9) is proportional to $\varepsilon$ ); as the latency differences decrease, $\varepsilon[k]$ could be decreased as well to drive the system to a smaller set $x_{\mathcal{W}}^{\varepsilon[k], \delta}$ that better approximates the Wardrop equilibrium. A control law with a decreasing $\varepsilon[k]$ is proposed and discussed in [25] for the single-commodity delay-free case, and is extended in this section to multi-commodity problems, also in the presence of unknown, time-varying but bounded, delays.

In our multi-commodity setup, if no information is shared among the commodities, only a heuristic law for the $\varepsilon[k]$ dynamics could be proposed: this law should be accurately tuned since, in practice, a too fast decay rate of $\varepsilon[k]$ would decrease the control gain too early, affecting the algorithm convergence speed. Conversely, in this section, we assume that the commodities may exchange some information and we define a time-varying tolerance $\varepsilon[k]$ such that the system trajectories $\boldsymbol{x}[k]$ asymptotically tend to $\mathcal{X}_{\mathcal{W}}^{0, \delta}$, i.e., such that the latencies of the $\delta$-loaded paths are asymptotically equalised.

The time-varying control law is then modified by considering time-varying tolerance and gain in (7) and (9), respectively:

$$
\begin{aligned}
& \mu_{p q}^{i}[k]=\left\{\begin{array}{l}
0 \text { if } l_{p}\left[k-h_{p}^{i}[k]\right]-l_{q}\left[k-h_{q}^{i}[k]\right] \leq \varepsilon[k], \\
1 \text { otherwise }
\end{array}\right. \\
& \sigma_{p q}^{i}[k]=\frac{\sigma[k]}{\tau d^{i} \bar{\beta}^{i}\left(\left|\mathcal{P}^{i}\right|+\eta^{i}\right)|\mathcal{C}|(1+\bar{h})} .
\end{aligned}
$$

The properties of the proposed tolerance and control gain are summarised in Assumption 2.

Assumption 2. The time-varying tolerance $\varepsilon[k]$ and the timevarying gain $\sigma[k]$ have the following properties:

A2.1 $\varepsilon[-h]=\varepsilon_{0}$ with $0<\varepsilon_{0} \leq \min _{i \in \mathcal{C}} \bar{\beta}^{i} d^{i}$ for all $h \in \mathcal{H}$;

A2.2 $\varepsilon[k+1] \leq \varepsilon[k], k=0,1, \ldots$ and $\lim _{k \rightarrow \infty} \varepsilon[k]=0$;

A2.3 $\sigma[k-\bar{h}] \leq \varepsilon[k], k=-\bar{h},-\bar{h}+1, \ldots$.

We note that A2.1 has the same meaning of equation (8), that A2.2 states that the tolerance must be non-increasing and must asymptotically converge to 0 and that A2.3 limits the control gain to a future value of the tolerance. The control law described later in this section will propose a way to set the tolerance and the gain according to Assumption 2, under which the following convergence result holds.

Theorem 4. Under Assumptions 1 and 2, considering the control law (6), (23) and (24), the ASR dynamics robustly asymptotically converges to a $(0, \delta)$-Wardrop equilibrium under heterogeneous, time-varying, unknown but bounded delays $\boldsymbol{h}[k] \in \mathcal{H}^{\mathcal{C} \times \mathcal{P}}$.

\section{Proof (see Appendix 1).}

In order to obtain a control law which can be enforced in practice, the assumption is that each commodity (e.g., the source node of each commodity) communicates to the other ones its current measure of the maximum measured latency mismatch among the $\delta$-loaded paths, computed as

$$
e_{\delta}^{i}[k]=\max _{p \in \mathcal{P}^{i} \mid x_{p}^{i}[k]>\delta} l_{p}\left[k-h_{p}^{i}[k]\right]-\min _{p \in \mathcal{P} i} l_{p}\left[k-h_{q}^{i}[k]\right]
$$

Then, at time $k$, all the commodities can compute a common non-increasing tolerance upper-bound as

$\bar{e}_{\delta}[k]=\left\{\begin{array}{l}\varepsilon_{0} \text { if } k \leq \bar{h} \\ \min \left\{\bar{e}_{\delta}[k-1], \max _{i \in \mathcal{C}} e_{\delta}^{i}[k-1]\right\} \text { otherwise }\end{array}\right.$

with $\varepsilon_{0}$ chosen as in equation (8), i.e., $0<\varepsilon_{0} \leq \min _{i \in \mathcal{C}} \bar{\beta}^{i} d^{i}$. Note that, due to equation (26), $\delta$ is now an additional controller design parameter.

In [24], a time-varying tolerance law is proposed for the single-commodity delay-free case, where $\varepsilon[k]$ is proportional, by a parameter $0<\alpha<1$, to the current maximum latency mismatch. Taking inspiration from that idea, the proposed timevarying tolerance law and the controller gain are defined as $\varepsilon[k]=\alpha \bar{e}_{\delta}[k-\bar{h}]$,

$\sigma[k]=\alpha \bar{e}_{\delta}[k]$

respectively, where the parameter $0<\alpha<1$ sets a trade-off between the gain value and the number of flows selected for 
migrations according to equation $(23)^{3}$.

Assumption A2.3 holds since $\sigma[k]=\varepsilon[k+\bar{h}]$. The nonincreasing sequence (27) is also such that the assumption A2.2 holds. In fact, let us assume that there exists a number $k^{\prime}$ such that $\bar{e}_{\delta}[k]$ is constant for all $k \geq k^{\prime}$. Let us define such a constant with $\bar{e}_{\delta}^{\prime}$. In this case, both (27) and (28) are constant for all $k \geq k^{\prime}+\bar{h}$, with $\varepsilon[k]=\alpha \bar{e}_{\delta}^{\prime}$ and $\sigma[k]=\alpha \bar{e}_{\delta}^{\prime}$, and Theorem 3 holds. Hence, the system trajectories asymptotically tend to the set $x_{w}^{\alpha \bar{e}_{\delta}^{\prime} \delta}$, defined as the set where the latency mismatch among the $\delta$-loaded paths is at most $\alpha \bar{e}_{\delta}^{\prime}$, i.e., where $e_{\delta}^{i}[k] \leq \alpha \bar{e}_{\delta}^{\prime}$ for all $i \in \mathcal{C}$. Therefore, since $\alpha<1$, the error $e_{\delta}^{i}[k]$ becomes smaller than $\bar{e}_{\delta}^{\prime}$ in finite time, for all $i \in \mathcal{C}$, which, in turn, decreases $\bar{e}_{\delta}[k]$ below $\bar{e}_{\delta}^{\prime}$ and, hence, $\varepsilon[k]$ below $\alpha \bar{e}_{\delta}^{\prime}$, contradicting the assumption.

Remark 1. In practice, initially the algorithm keeps $\varepsilon[k]=\varepsilon_{0}$ for $\bar{h}$ time-steps and the system behaves as with the algorithm of Section V. As $\varepsilon[k]$ starts decreasing, the system trajectories are attracted by the sequence of sets $\left\{x_{\mathcal{W}}^{\varepsilon[k], \delta}\right\}_{k=0,1, \ldots}$, which shrink with $k$. The key is the migration policy (23): i) at any time step $k$, equation (23) sets a minimum latency separation $\varepsilon[k]$ for the migration between two paths; (ii) such latency separation decreases with $k$, as the latency values converge.

\section{NUMERICAL SimULATIONS}

In this section, the proposed algorithm is evaluated via numerical simulations performed with MATLAB $®$. The example scenario, depicted in Fig. 1, has $|\mathcal{C}|=2$ commodities, $|\mathcal{E}|=17$ edges and $|\mathcal{P}|=17$ paths. $\left|\mathcal{P}^{1}\right|=11$ and $\left|\mathcal{P}^{2}\right|=6$ paths are available to commodity 1 and 2 , respectively. The paths, listed in Table 1, support the total traffic $d=1$, with $d^{1}=0.8$ and $d^{2}=0.2$, and are such that $\eta^{1}=6$ and $\eta^{2}=4$.

In the example, the sample time is $\tau=0.1 \mathrm{~s}$ and the latency functions are assumed to be the path delays. For simulation purposes, we implemented the following functions modelling, for each edge, how the delay increases with the load:

$l_{e}\left(x_{e}\right)=e^{a_{e} x_{e}}-1$,

with $a_{e}=0.45 \mathrm{~s}$, for $e \leq 5, a_{e}=0.7 \mathrm{~s}$ for $6 \leq e \leq 9, a_{e}=$ $0.25 s$ for $e \geq 10$. The maximum derivatives of the edge latency functions are computed as $a_{e} e^{a_{e} x_{e}}, e=1, \ldots, 17$. Considering the defined latency functions, the traffic demand and the considered paths, the maximum path latency is bounded by $2.24 s$ and we obtain $\bar{\beta}^{1}=3.07$ and $\bar{\beta}^{2}=2.64$.

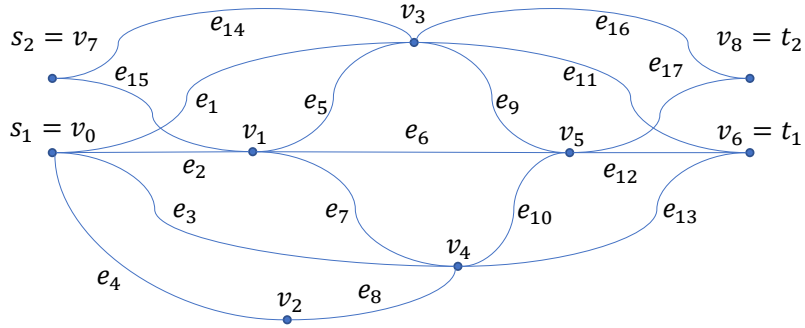

Figure 1. Example network $\left(v_{k}\right.$ : node $k ; s_{i}$ : source node of commodity $i ; t_{i}$ : destination node of commodity $i$; $e_{k}$ : edge $k$ ).

TABLE I

PATHS

\begin{tabular}{|c|c|}
\hline Paths of commodity 1 & Paths of commodity 2 \\
\hline $\begin{array}{l}p_{1}=\left\{e_{1}, e_{11}\right\} \\
p_{2}=\left\{e_{1}, e_{9}, e_{12}\right\} \\
p_{3}=\left\{e_{2}, e_{5}, e_{11}\right\} \\
p_{4}=\left\{e_{2}, e_{5}, e_{9}, e_{12}\right\} \\
p_{5}=\left\{e_{2}, e_{6}, e_{12}\right\} \\
p_{6}=\left\{e_{2}, e_{7}, e_{10}, e_{12}\right\} \\
p_{7}=\left\{e_{2}, e_{7}, e_{13}\right\} \\
p_{8}=\left\{e_{3}, e_{10}, e_{12}\right\} \\
p_{9}=\left\{e_{3}, e_{13}\right\} \\
p_{10}=\left\{e_{4}, e_{8}, e_{10}, e_{12}\right\} \\
p_{11}=\left\{e_{4}, e_{8}, e_{13}\right\}\end{array}$ & $\begin{array}{l}p_{12}=\left\{e_{14}, e_{16}\right\} \\
p_{13}=\left\{e_{14}, e_{9}, e_{17}\right\} \\
p_{14}=\left\{e_{15}, e_{5}, e_{16}\right\} \\
p_{15}=\left\{e_{15}, e_{5}, e_{9}, e_{17}\right\} \\
p_{16}=\left\{e_{15}, e_{6}, e_{17}\right\} \\
p_{17}=\left\{e_{15}, e_{7}, e_{10}, e_{17}\right\}\end{array}$ \\
\hline
\end{tabular}

Since, in this example, the latency functions are the path delays themselves, the delays are computed in sample times as:

$$
h_{p}^{i}[k]=\left[\tau \cdot l_{p}[k]\right], i=1,2, p=1, \ldots, 18, k \geq 0,
$$

yielding time-varying values upper-bounded by $\bar{h}=20$.

Fig. 2 and Fig. 3 show the dynamics of the latency and population values obtained with the algorithm with fixed tolerance, with $\varepsilon=0.02$, and with the algorithm with dynamic tolerance, with $\varepsilon_{0}=0.15$ and $\alpha=0.45$, with the same value for $\delta=2 \cdot 10^{-3}$. The two algorithms were simulated starting from the same randomly selected initial population.

Fig. 2 shows that the maximum latency mismatch among $\delta$ loaded paths, i.e., $\max \left\{e_{\delta}^{1}[k], e_{\delta}^{2}[k]\right\}, \quad$ asymptotically approaches $\varepsilon$. We observe that, after time $k=611$ (i.e., 61.1s), $e_{\delta}^{2}[k]$ has a sudden drop. This is due to the fact that the population $x_{16}^{2}[k]$ of path 16 , which is the path of commodity 2 characterised by the highest latency, is decreasing and, at $k=$ 612 , becomes smaller than $\delta$. From that time on, the path 16 is not a $\delta$-loaded path any longer and, thus, its latency $l_{16}[k]$ is no more considered in the computation of $e_{\delta}^{2}[k]$. Since the latency of path 16 was the highest one, $e_{\delta}^{2}[k]$ instantaneously decreases to a smaller value. The figure shows that also the population of path 18 becomes smaller than $\delta$ (at time $k=516$, with no visible effect on $e_{\delta}^{2}[k]$ since, at that time, path 16 was still a $\delta$ loaded one). In compliance with the $(\varepsilon, \delta)$-Wardrop equilibrium definition, the latencies of paths 16 and 18 , which are not $\delta$ loaded, are not equalised to the latencies of the other paths and remain higher.

case, which is still meaningful in the multi-commodity case for the proposed controller.
${ }^{3}$ Concerning the effects of the value of $\alpha$ on the algorithm convergence, the
reader is referred to the analysis in [24] for the single-commodity delay-free 

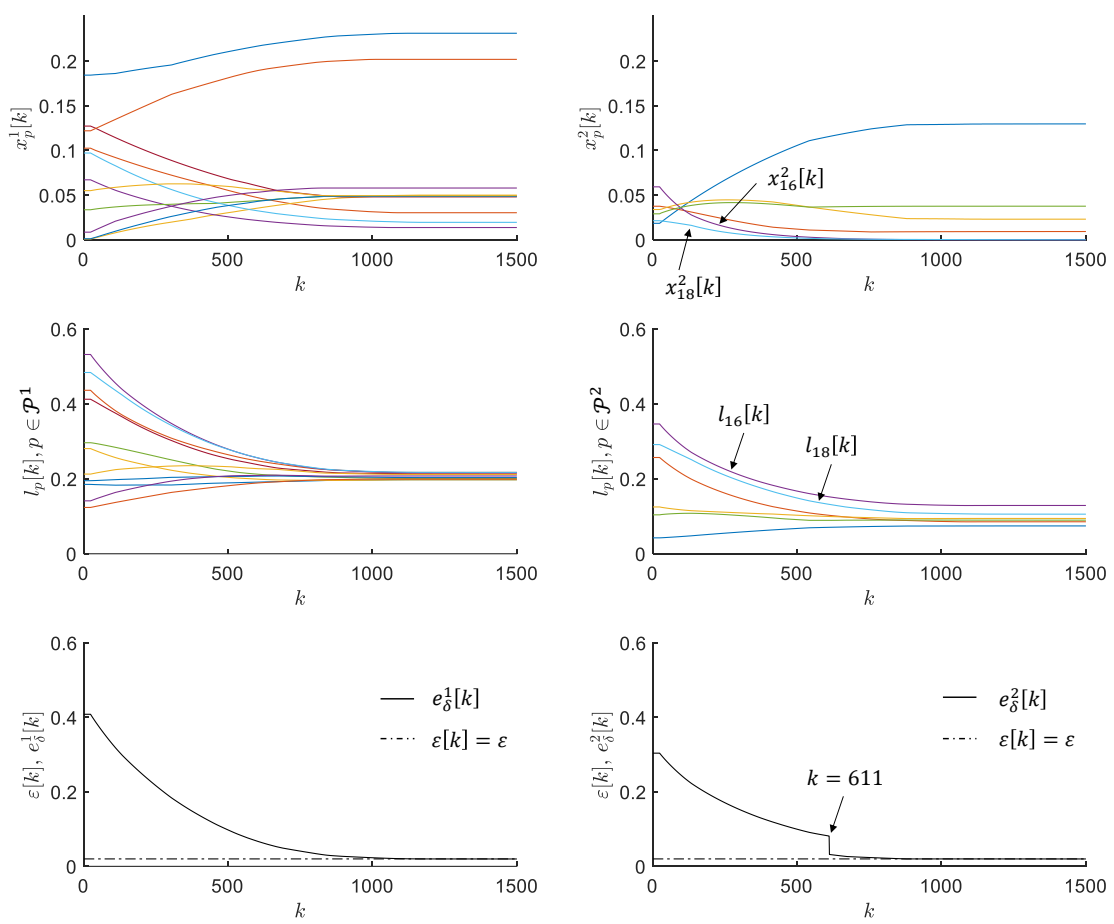

Figure 2. Population dynamics (higher plot), latency dynamics (middle plot) and latency mismatch (lower plot) with static tolerance $(\varepsilon=0.02)$.
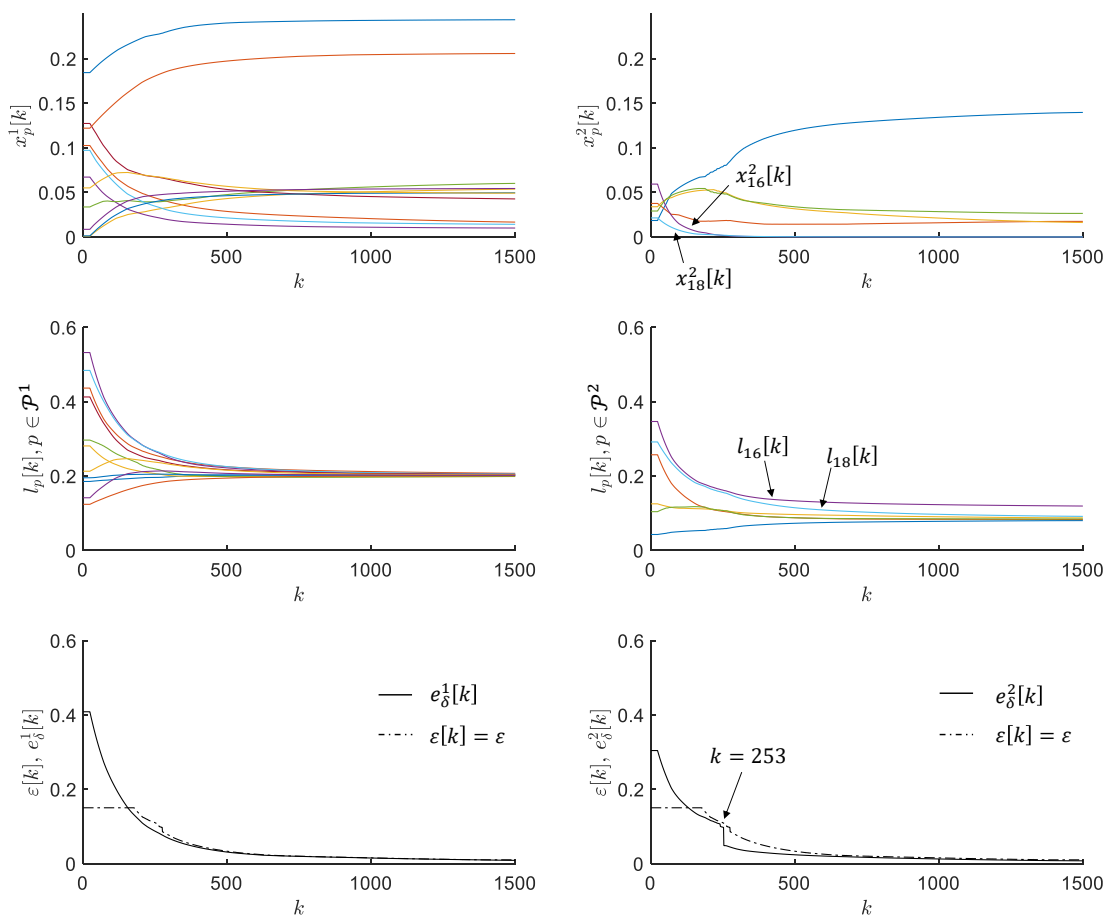

Figure 3. Population dynamics (higher plot), latency dynamics (middle plot) and latency mismatch (lower plot) with dynamic tolerance $\left(\varepsilon_{0}=0.15\right)$.

Analogous comments hold for the algorithm with dynamic tolerance. Fig. 3 highlights that the advantages of this approach are that i) initially, the tolerance $\varepsilon_{0}$ can be set to a large value to set a high gain $\sigma[k]$ and, thus, to achieve faster convergence, and ii) as the errors $e_{\delta}^{i}$ 's decrease, $\varepsilon[k]$ is decreased according to (27) to shrink the convergence set $\mathcal{X}_{\mathcal{W}}^{\varepsilon[k], \delta}$. To compare the performance of the two algorithms, we note that the maximum latency mismatch becomes smaller than 0.02 (which is the asymptotic convergence value set for the first algorithm) from $k=739$ on.

Fig. 3 clarifies the need of introducing the parameter $\delta$ in the definition of the approximate Wardrop equilibrium (4). If only the paths with no load were considered as unloaded paths, path 16 would have been considered as always loaded (its population $x_{16}^{2}[k]$ goes to 0 asymptotically), its latency $l_{16}[k]$ would be 
always considered in the computation of $e_{\delta}^{2}[k]$ (according to equation (25), with $\delta=0$ ) and, therefore, $\varepsilon[k]$ would not asymptotically annihilate.

\section{CONCLUSIONS AND FUTURE WORK}

This paper presented a discrete-time, distributed and noncooperative algorithm for multi-commodity routing problems, that was proven to converge to an arbitrarily small neighbourhood of the Wardrop equilibrium even in the presence of heterogeneous, unknown, time-varying but bounded communication delays. The paper discussed also how the algorithm can be improved in the case in which each commodity is provided with some information also on the network paths used by the other commodities, obtaining a better reconstruction of the Wardrop equilibrium.

Future work is aimed at extending the results of the paper in the multi-rate system domain, assuming hence different decision instants for the various agents, and time-varying networks, in which both the latency functions and the network topology may evolve over time.

\section{APPENDIX 1}

Proof of Lemma 1. Considering the generic edge $e \in p$ at time $k$, the maximum latency decrease occurs when no commodities migrate their populations from the paths not including $e$ to the paths including $e$ :

$l_{e}\left(x_{e}[k+1]\right)$

$=l_{e}\left(x_{e}[k]+\tau \sum_{p \in \mathcal{P}_{e}} \sum_{m \in \mathcal{P} \backslash \mathcal{P}_{e}}\left(r_{m p}[k]-r_{p m}[k]\right)\right)$

$\geq l_{e}\left(x_{e}[k]-\tau \sum_{p \in \mathcal{P}_{e}} \sum_{m \in \mathcal{P} \backslash \mathcal{P}_{e}}\left(r_{p m}[k]\right)\right)$.

Since $\beta_{e}$ is the Lipschitz constant of the function $l_{e}$, it follows that

$l_{e}\left(x_{e}[k+1]\right) \geq l_{e}\left(x_{e}[k]\right)-\tau \beta_{e} \sum_{p \in \mathcal{P}_{e}} \sum_{m \in \mathcal{P} \backslash \mathcal{P}_{e}} r_{p m}[k]$.

Considering equations (5), (7) and (9), the last term of equation (A2) is written as

$\tau \beta_{e} \sum_{p \in \mathcal{P}_{e}} \sum_{m \in \mathcal{P} \backslash \mathcal{P}_{e}} r_{p m}[k]=\tau \beta_{e} \sum_{i \in C} \sum_{p \in \mathcal{P}_{e}^{i}} \sum_{m \in \mathcal{P}^{i} \backslash \mathcal{P}_{e}^{i}} r_{p m}^{i}[k]$

$=\sum_{i \in C} \sum_{p \in \mathcal{P}_{e}^{i}} \tau \beta_{e} \sum_{m \in \mathcal{P}^{i} \backslash \mathcal{P}_{e}^{i}} x_{p}^{i}[k] \sigma_{p m}^{i}[k] \mu_{p m}^{i}[k]$

$\leq \sum_{i \in C} \frac{\beta_{e} \sigma}{d^{i} \bar{\beta}^{i}|C|\left(\left|\mathcal{P}^{i}\right|+\eta^{i}\right)(1+\bar{h})} \sum_{m \in \mathcal{P}^{i} \backslash \mathcal{P}_{e}^{i}} x_{p}^{i}[k] \sum_{p \in \mathcal{P}_{e}^{i}} \mu_{p m}^{i}[k]$,

where the inequality holds since $\beta_{e} \leq \bar{\beta}^{i}$ for all $i$ such that $\mathcal{P}_{e}^{i} \neq$ $\emptyset$. Since there are at most $\eta^{i}$ terms in the last summation of equation (A3) and since $\sum_{p \in \mathcal{P}_{e}} x_{p}^{i}[k] \leq d^{i}$, it holds that

$\tau \beta_{e} \sum_{p \in \mathcal{P} \mid e \in p} \sum_{m \in \mathcal{P} \mid e \notin p} r_{p m}[k] \leq \sum_{i \in C} \frac{\beta_{e} \sigma}{\bar{\beta}^{i}|C|(1+\bar{h})} \frac{\eta^{i}}{\left|\mathcal{P}^{i}\right|+\eta^{i}}$.

Given equations (A1) and (A4) and recalling that $l_{p}=$ $\sum_{e \in p} l_{e}$ and $\bar{\beta}^{i} \geq \sum_{e \in p} \beta_{e}$ for all paths $p \in \mathcal{P}^{i} i$, it holds that

$l_{p}[k+1] \geq \sum_{e \in p}\left(l_{e}[k]-\sum_{i \in C} \frac{\beta_{e} \sigma}{\bar{\beta}^{i}|C|(1+\bar{h})} \frac{\eta^{i}}{\left|\mathcal{P}^{i}\right|+\eta^{i}}\right)$

$$
\begin{aligned}
& =l_{p}[k]-\frac{\sigma}{|C|(1+\bar{h})} \sum_{i \in C} \frac{1}{\bar{\beta}^{i}} \frac{\eta^{i}}{\left|\mathcal{P}^{i}\right|+\eta^{i}} \sum_{e \in p} \beta_{e} \\
& \geq l_{p}[k]-\frac{\sigma}{|C|(1+\bar{h})} \sum_{i \in C} \frac{\eta^{i}}{\left|\mathcal{P}^{i}\right|+\eta^{i}}
\end{aligned}
$$

Similarly, the maximum latency increase occurs when no commodities migrate their populations from paths including $e$ to paths not including $e$ :

$l_{e}\left(x_{e}[k+1]\right) \leq l_{e}\left(x_{e}[k]\right)+\tau \beta_{e} \sum_{m \in \mathcal{P} \backslash \mathcal{P}_{e}} \sum_{p \in \mathcal{P}_{e}} r_{m p}[k]$.

Considering equations (5), (7) and (9), the last term of equation (A6) is written as

$\tau \beta_{e} \sum_{m \in \mathcal{P} \backslash \mathcal{P}_{e}} \sum_{p \in \mathcal{P}_{e}} r_{m p}[k]=\tau \beta_{e} \sum_{i \in C} \sum_{m \in \mathcal{P}_{e}^{i}} \sum_{p \in \mathcal{P} i} \backslash \mathcal{P}_{e}^{i} r_{m p}^{i}[k]$

$=\sum_{i \in C} \sum_{m \in \mathcal{P}_{e}^{i}} \tau \beta_{e} \sum_{p \in \mathcal{P}^{i} \backslash \mathcal{P}_{e}^{i}} x_{m}^{i}[k] \sigma_{m p}^{i}[k] \mu_{m p}^{i}[k]$

$\leq \sum_{i \in C} \frac{\beta_{e} \sigma}{\bar{\beta}^{i} d^{i}|C|\left(\left|\mathcal{P}^{i}\right|+\eta^{i}\right)(1+\bar{h})} \sum_{m \in \mathcal{P}_{e}^{i}} x_{m}^{i}[k] \sum_{p \in \mathcal{P} i \mathcal{P}_{e}^{i}} \mu_{m p}^{i}[k]$,

Since there are at most $\left|\mathcal{P}^{i}\right|$ terms in the last summation of equation (A7) it holds that

$\tau \beta_{e} \sum_{m \in \mathcal{P} \backslash \mathcal{P}_{e}} \sum_{p \in \mathcal{P}_{e}} r_{m p}[k] \leq \sum_{i \in C} \frac{\beta_{e} \sigma}{\bar{\beta}^{i}|C|(1+\bar{h})} \frac{\left|\mathcal{P}^{i}\right|}{\left|\mathcal{P}^{i}\right|+\eta^{i}}$.

Given equations (A6) and (A8) it follows that

$l_{p}[k+1] \leq \sum_{e \in p}\left(l_{e}[k]+\sum_{i \in C} \frac{\beta_{e} \sigma}{\bar{\beta}^{i}|C|(1+\bar{h})} \frac{\left|\mathcal{P}^{i}\right|}{\left|\mathcal{P}^{i}\right|+\eta^{i}}\right)$

$\leq l_{p}[k]+\frac{\sigma}{|C|(1+\bar{h})} \sum_{i \in C} \frac{\left|\mathcal{P}^{i}\right|}{\left|\mathcal{P}^{i}\right|+\eta^{i}}$

Equation (12) follows from equations (A5) and (A9).

Proof of Lemma 2. It is shown in the following that, if the initial flow vector of the considered system dynamics, $\boldsymbol{z}[0]$, lies in $Z$, the flow vector $\boldsymbol{Z}[k]$ lies in $Z$ as well. Recalling equations (15) and (18), it is sufficient to show that a) $\sum_{p \in \mathcal{P} i} x_{p}^{i}[k]=d^{i}$ and b) $x_{p}^{i}[k] \geq 0, \forall p \in \mathcal{P}^{i}, \forall i \in \mathcal{C}, \forall k \geq 0$.

a) It follows from equation (5) that, for all $i \in \mathcal{C}$ and $s \geq 0$,

$$
\begin{aligned}
& \sum_{p \in \mathcal{P}^{i}}\left(x_{p}^{i}[k+1]-x_{p}^{i}[k]\right)=\tau \sum_{p \in \mathcal{P}^{i}} \sum_{q \in \mathcal{P}^{i}}\left(r_{q p}^{i}[k]-r_{p q}^{i}[k]\right) \\
& =\tau\left(\sum_{p \in \mathcal{P}^{i}} \sum_{q \in \mathcal{P}^{i}} r_{q p}^{i}[k]-\sum_{q \in \mathcal{P}^{i}} \sum_{p \in \mathcal{P}^{i}} r_{q p}^{i}[k]\right)=0,
\end{aligned}
$$

and, therefore, that $\sum_{p \in \mathcal{P}^{i}} x_{p}^{i}[k]=\sum_{p \in \mathcal{P}^{i}} x_{p}^{i}[0]=d^{i}, \forall k \geq 0$.

b) Given that equation (11) yields $x_{p}^{i}[-h] \geq 0, \forall h \in \mathcal{H}$, it is proven below by induction that $x_{p}^{i}[k] \geq 0, \forall k \geq 0$. Assuming that $x_{p}^{i}[k] \geq 0$, for a given $k$, it is sufficient to prove that

$x_{p}^{i}[k+1]=x_{p}^{i}[k]+\tau \sum_{q \in \mathcal{P}}\left(r_{q p}^{i}[k]-r_{p q}^{i}[k]\right) \geq 0, \forall p \in$ $\mathcal{P}^{i}, \forall i \in \mathcal{C}$.

If $x_{p}^{i}[k]=0$, it follows that $r_{p q}^{i}[k]=0$ and thus equation (A11) yields $x_{p}^{i}[k+1] \geq 0$. 
If $x_{p}^{i}[k]>0$, from equation (5) it follows that $r_{p q}^{i}[k] \geq 0$. Thus, the following inequality holds (in the worst case, no paths migrate part of their population to a path $p$ ):

$x_{p}^{i}[k+1] \geq x_{p}^{i}[k]-\tau \sum_{q \in \mathcal{P}^{i}} r_{p q}^{i}[k]$.

A sufficient condition for inequality (A11) to hold is then

$x_{p}^{i}[k]-\tau \sum_{q \in \mathcal{P}^{i}} r_{p q}^{i}[k] \geq 0$.

Recalling equations (6) and (9), equation (A13) is written as

$x_{p}^{i}[k]-\tau \sum_{q \in \mathcal{P}^{i}} x_{p}^{i}[k] \sigma_{p q}^{i}[k] \mu_{p q}^{i}[k]$

$=x_{p}^{i}[k]\left(1-\tau \sum_{q \in \mathcal{P} i} \sigma_{p q}^{i}[k] \mu_{p q}^{i}[k]\right)$

$\geq x_{p}^{i}[k]\left(1-\frac{\sigma}{\bar{\beta}^{i} d^{i}|\mathcal{C}|\left(\left|\mathcal{P}^{i}\right|+\eta^{i}\right)(1+\bar{h})} \sum_{q \in \mathcal{P}^{i}} \mu_{p q}^{i}[k]\right)$

$>x_{p}^{i}[k]\left(1-\frac{\sigma}{\bar{\beta}^{i} d^{i}|\mathcal{C}|(1+\bar{h})}\right) \geq 0$,

where the second inequality holds since the summation has at most $\left|\mathcal{P}^{i}\right|$ terms equal to 1 . In the case $x_{p}^{i}[k]>0$, equations (8), (10) are sufficient for (A14) to hold.

Proof of Lemma 3. From Property 1, it holds that $\mathcal{L}(\mathbf{z})$ is positive definite.

We need to prove that $\Delta \mathcal{L}(\mathbf{z}[k])$ is negative semi-definite. The definition (22) of $\Delta \mathcal{L}(\boldsymbol{z}[k])$ yields

$\Delta \mathcal{L}(\mathbf{z}[k])=\sum_{h \in \mathcal{H}}\left(\Phi\left(\mathbf{z}_{h}[k+1]\right)-\Phi\left(\mathbf{z}_{h}[k]\right)\right)$

$=\sum_{h \in \mathcal{H}} \sum_{e \in \mathcal{E}} \int_{x_{e}[k-h]}^{x_{e}[k+1-h]} l_{e}(\xi) d \xi$

$\leq \sum_{h \in \mathcal{H}} \sum_{e \in \mathcal{E}}\left(x_{e}[k+1-h]-x_{e}[k-h]\right) l_{e}\left(x_{e}[k+1-h]\right)$,

(A15)

where the inequality holds from geometric considerations, as the latency functions are strictly increasing (see Appendix 2).

Considering that

$\sum_{e \in \mathcal{E}}\left(x_{e}[k+1-h]-x_{e}[k-h]\right) l_{e}\left(x_{e}[k+1-h]\right)$

$=\sum_{e \in \mathcal{E}} \sum_{p \in \mathcal{P}_{e}}\left(x_{p}[k+1-h]-x_{p}[k-h]\right) l_{e}\left(x_{e}[k+1-h]\right)$

$=\sum_{p \in \mathcal{P}} \sum_{e \in p}\left(x_{p}[k+1-h]-x_{p}[k-h]\right) l_{e}\left(x_{e}[k+1-h]\right)$

$=\sum_{p \in \mathcal{P}}\left(x_{p}[k+1-h]-x_{p}[k-h]\right) l_{p}[k+1-h]$,

(A16)

from equations (A15) and (A16), it follows that

$\Delta \mathcal{L}(\mathbf{z}[k]) \leq \sum_{h \in \mathcal{H}} \sum_{p \in \mathcal{P}}\left(x_{p}[k+1-h]-x_{p}[k-h]\right) l_{p}[k+$ $1-h]$.

Let us consider the inner summation of (A17) for a given $h \in$ $\mathcal{H}$ :

$\sum_{p \in \mathcal{P}}\left(x_{p}[k+1-h]-x_{p}[k-h]\right) l_{p}[k+1-h]$

$=\tau \sum_{p \in \mathcal{P}}\left(\sum_{q \in \mathcal{P}} r_{q p}[k-h]-\sum_{q \in \mathcal{P}} r_{p q}[k-h]\right) l_{p}[k+1-h]$

$=\tau \sum_{p \in \mathcal{P}} \sum_{q \in \mathcal{P}} r_{q p}[k-h] l_{p}[k+1-h]-$

$\tau \sum_{p \in \mathcal{P}} \sum_{q \in \mathcal{P}} r_{p q}[k-h] l_{p}[k+1-h]$

$=\tau \sum_{q \in \mathcal{P}} \sum_{p \in \mathcal{P}} r_{p q}[k-h] l_{q}[k+1-h]-$ $\tau \sum_{p \in \mathcal{P}} \sum_{q \in \mathcal{P}} r_{p q}[k-h] l_{p}[k+1-h]$

$=\tau \sum_{p \in \mathcal{P}} \sum_{q \in \mathcal{P}} r_{p q}[k-h]\left(l_{q}[k+1-h]-l_{p}[k+1-h]\right)$.

We now prove that the terms

$$
r_{p q}[k-h]\left(l_{q}[k+1-h]-l_{p}[k+1-h]\right)
$$

of the last summation are either negative or null, $\forall p, q \in \mathcal{P}$.

a) If $r_{p q}[k-h]=0$, the term is null.

b) It is shown below that, if $r_{p q}[k-h]>0$, it holds that $l_{p}[k+1-h]-l_{q}[k+1-h]>0$, i.e., the considered term in (A18) is negative.

Since we are considering the case $r_{p q}[k-h]>0$, it holds from equations (6), (7) that

$l_{p}\left[k-h-h_{p}^{i}[k-h]\right]-l_{q}\left[k-h-h_{q}^{i}[k-h]\right]>\varepsilon$,

at least for one $i \in \mathcal{C}$.

A useful result obtained by iteratively using Lemma 1 is that

$l_{p}[k-h+1]-l_{q}[k-h+1] \geq\left(l_{p}\left[k-h-h_{p}^{i}[k-h]\right]-\right.$

$\left.\left(1+h_{p}^{i}[k-h]\right) \frac{\sigma}{|\mathcal{C}|(1+\bar{h})} \sum_{i \in C} \frac{\eta^{i}}{\left|\mathcal{P}^{i}\right|+\eta^{i}}\right)-\left(l_{q}\left[k-h-h_{q}^{i}[k-\right.\right.$

$\left.h]]+\left(1+h_{q}^{i}[k-h]\right) \frac{\sigma}{|\mathcal{C}|(1+\bar{h})} \sum_{i \in \mathcal{C}} \frac{\left|\mathcal{P}^{i}\right|}{\left|\mathcal{P}^{i}\right|+\eta^{i}}\right)$.

From equation (A20) and equation (13), given that $h_{p}^{i}[k] \leq$ $\bar{h}, \forall p \in \mathcal{P}, k=-\bar{h},-\bar{h}+1, \ldots$, it follows that

$l_{p}[k-h+1]-l_{q}[k-h+1]$

$\geq\left(l_{p}\left[k-h-h_{p}^{i}[k-h]\right]-\frac{\sigma}{|\mathcal{C}|} \sum_{i \in \mathcal{C}} \frac{\eta^{i}}{|\mathcal{P} i|+\eta^{i}}\right)$

$-\left(l_{q}\left[k-\mathrm{h}-h_{q}^{i}[k-h]\right]+\frac{\sigma}{|\mathcal{C}|} \sum_{i \in \mathcal{C}} \frac{\left|\mathcal{P}^{i}\right|}{\left|\mathcal{P}^{i}\right|+\eta^{i}}\right)$

$\geq l_{p}\left[k-h-h_{q}^{i}[k-h]\right]-l_{q}\left[k-h-h_{q}^{i}[k-h]\right]-\sigma$.

From equation (10), (A19) and (A21) it follows that

$l_{p}[k+1-h]-l_{q}[k+1-h]>\varepsilon-\sigma \geq 0$,

Proof of Theorem 3. Thanks to the LaSalle's invariance principle (Theorem 1), we know that the system trajectories asymptotically tend to the maximal invariant set $\mathcal{M}$ in which $\Delta \mathcal{L}(\mathbf{z})=0$, where $\mathcal{L}(\mathbf{z})$ is the Lyapunov function (21) (see Lemma 3 ). We will show below that, in our case, the set $\mathcal{M}$ can be expressed as

$\mathcal{M}:=\left\{\mathbf{z} \in \mathcal{Z} \mid \mathbf{z}_{h}=\mathbf{z}_{0}, \forall h \in \mathcal{H}\right.$ and $l_{p}\left(\mathbf{z}_{0}\right)-l_{q}\left(\mathbf{z}_{0}\right)<$

$\varepsilon, \forall p, q \in \mathcal{P}^{i}$ s.t. $\left.z_{p 0}^{i}>\delta, \forall i \in \mathcal{C}\right\}$.

(A23) 
Firstly, we note that, by definition, $\mathcal{M} \subset\left(\chi_{\mathcal{W}}^{\varepsilon, \delta}\right)^{\bar{h}}$ and, hence, that $\mathcal{M} \neq \varnothing$ since $\mathcal{Z}_{\mathcal{W}}^{\varepsilon, \delta}$ contains at least $\boldsymbol{z}_{\mathcal{W}}$.

We show now that the set (A23) is positively invariant and $\Delta \mathcal{L}(\boldsymbol{z})=0$ for all $\boldsymbol{z} \in \mathcal{M}$. Let us suppose that $\boldsymbol{z}[0]=\boldsymbol{z} \in$ $\mathcal{M}$. By the definition (A23), it holds that 1) $r_{p q}^{i}[0]=0, \forall p, q \in$ $\mathcal{P}, \forall i \in \mathcal{C}$, implying that $\left.\Delta L\left(\boldsymbol{z}_{0}[0]\right)=0 ; 2\right) \boldsymbol{z}_{h}[0]=\boldsymbol{z}_{h-1}[0]$ and hence $\Delta L\left(z_{h}[0]\right)=0, \forall h \in \mathcal{H} \backslash\{0\}$. It follows that $\Delta \mathcal{L}(\boldsymbol{z}[0])=0$. Moreover, since $r_{p q}^{i}[0]=0$, it holds that $\boldsymbol{z}_{0}[1]=\boldsymbol{z}_{0}$ [0], which, given the dynamics (20), implies $\boldsymbol{z}[1]=\boldsymbol{z}[0] \in \mathcal{M}$, meaning that $\mathcal{M}$ is positively invariant.

Now we prove that if $\boldsymbol{z}[0] \notin \mathcal{M}$ then either $\Delta \mathcal{L}(\boldsymbol{z}[0])<0$ or $\boldsymbol{z}[k] \in \mathcal{M}$ for a given $k \leq \bar{h}+1$. The demonstration relies on the following iterative procedure:

Step 0 . Let $j=0$.

Step 1. The following 2 cases are possible:

Case 1. $z[j] \in \mathcal{M}$ (q.e.d.).

Case 2. $z[j] \notin \mathcal{M}$; the following 2 cases are possible:

Case 2.1. There exist at least one number $h \in \mathcal{H} \backslash\{0\}$ such that $\boldsymbol{z}_{h}[j] \neq \boldsymbol{z}_{h-1}[j]$ with $\Delta L\left(\boldsymbol{z}_{h}[j]\right)<0$ (q.e.d.).

Case 2.2. There exist at least one number $k \in \mathcal{H} \backslash\{0\}$ such that $\boldsymbol{z}_{k}[j] \neq \boldsymbol{z}_{k-1}[j]$ but $\Delta L\left(\boldsymbol{z}_{h}[j]\right)=0$ for all $h \in \mathcal{H} \backslash\{0\}$; then the following 2 cases are possible:

Case 2.2.1. There exist a pair $p, q \in \mathcal{P}^{i}$ for a commodity $i \in \mathcal{C}$ with $\mathbf{z}_{p 0}^{i}>\delta$ such that

$l_{p}\left(\boldsymbol{z}_{h_{p}^{i}[j]}[j]\right)-l_{q}\left(\boldsymbol{z}_{h_{q}^{i}[j]}[j]\right)>\varepsilon$,

implying $r_{p q}^{i}[j]>0$ and $\Delta L\left(z_{0}[j]\right)<0$ (q.e.d.).

Case 2.2.2. For all pairs $p, q \in \mathcal{P}^{i}$ and for the commodities $i \in$ $\mathcal{C}$ such that $\boldsymbol{z}_{p 0}^{i}[j]>\delta$, it holds that

$l_{p}\left(\boldsymbol{z}_{h_{p}^{i}[j]}[j]\right)-l_{q}\left(\boldsymbol{z}_{h_{q}^{i}[j]}[j]\right) \leq \varepsilon$

implying $r_{p q}^{i}[j]=0$, which, in turn, implies $\Delta L\left(\boldsymbol{z}_{0}[j]\right)=0$ and $z_{0}[j+1]=z_{0}[j]$. Note that, at the $j$-th iteration of Case 2.2 , the system dynamics (20) leads to

$\boldsymbol{z}_{0}[j+1]=\boldsymbol{z}_{0}[0]$;

$\boldsymbol{z}_{1}[j+1]=\boldsymbol{z}_{0}[0]$;

$\boldsymbol{z}_{j}[j+1]=\boldsymbol{z}_{0}[0]$

If $j<\bar{h}$, let $j=j+1$ and repeat the procedure from Step 1 .

Otherwise, if $j=\bar{h}$, it follows from (A25) that $\boldsymbol{z}_{h}[\bar{h}+1]=$ $\boldsymbol{z}_{0}[0], \forall h \in \mathcal{H}$, which, in turn, considering equation (A24), implies that, for all $p, q \in \mathcal{P}^{i}$ such that $\boldsymbol{z}_{p 0}^{i}>\delta$ and for all $i \in$ $\mathcal{C}$,

$$
\begin{gathered}
l_{p}\left(\boldsymbol{z}_{h_{p}^{i}[\bar{h}+1]}[\bar{h}+1]\right)-l_{q}\left(\mathbf{z}_{h_{q}^{i}[\bar{h}+1]}[\bar{h}+1]\right)= \\
=l_{p}\left(\mathbf{z}_{0}[\bar{h}+1]\right)-l_{q}\left(\mathbf{z}_{0}[\bar{h}+1]\right) \leq \varepsilon,
\end{gathered}
$$

and, therefore, that $\mathbf{z}[\bar{h}+1] \in \mathcal{M}$ (q.e.d.).

In order to apply the LaSalle's invariance principle, $\mathcal{M}$ must be the maximal invariant set, which contains all and only the invariant trajectories of the system.

We firstly show that all points in $\mathcal{M}$ are part of an invariant trajectory. Recalling that we have already proven that $\mathcal{M}$ is positively invariant, let $\boldsymbol{z}[0] \in \mathcal{M}$. The trajectory that stays identically in $\boldsymbol{z}[0]$, i.e., $\boldsymbol{z}[k]=\boldsymbol{z}[0], k=1,2, \ldots$, represents a valid evolution of the system that remains in $\mathcal{M}$ for all $k \in \mathbb{R}$.

Secondly, for the discussion regarding Case 2.2 of the iterative procedure, no other points for which $\Delta L(\mathbf{z})=0$ can stay identically outside of the set $\mathcal{M}$. Furthermore, no other invariant trajectory of interest for the system exists, as in any trajectory that does not stay identically in a point there must exist a pair $p, q \in \mathcal{P}^{i}$ and a commodity $i \in \mathcal{C}$ for which $r_{p q}^{i}[k]>0$, for some $k \in \mathbb{R}$. Since we have shown in the proof of Lemma 3 that $r_{p q}^{i}[k]>0$ implies $\Delta \mathcal{L}(\mathbf{z}[\boldsymbol{k}])<0$, this concludes the proof.

Proof of Theorem 4. The proof follows the same steps of the ones of Lemmata 1-3 and of Theorem 3, therefore here we will only specify the differences due to the time-varying tolerance and gain values. Specifically, the only key difference is that the gain $\sigma[k]$ is time-varying and, by assumption A2.2, nonincreasing, i.e.:

$$
\sigma[k] \leq \sigma\left[k-h_{p}^{i}[k]\right] \leq \sigma[k-\bar{h}], \forall p \in \mathcal{P}^{i}, \forall i \in \mathcal{C}
$$

From Lemma 1, it holds thus that

$$
\begin{aligned}
& l_{p}[k-h+1]-l_{q}[k-h+1] \geq\left(l_{p}\left[k-h-h_{p}^{i}[k-h]\right]-\right. \\
& \left.\left(1+h_{p}^{i}[k-h]\right) \frac{\sigma[k-h-\bar{h}]}{|\mathcal{C}|(1+\bar{h})} \sum_{i \in C} \frac{\eta^{i}}{\left|\mathcal{P}^{i}\right|+\eta^{i}}\right)+ \\
& -\left(l_{q}\left[k-h-h_{q}^{i}[k-h]\right]+\left(1+h_{q}^{i}[k-\right.\right. \\
& \left.h]) \frac{\sigma[k-h-\bar{h}]}{|\mathcal{C}|(1+\bar{h})} \sum_{i \in \mathcal{C}} \frac{\left|\mathcal{P}^{i}\right|}{\left|\mathcal{P}^{i}\right|+\eta^{i}}\right),
\end{aligned}
$$

for all $h \in \mathcal{H}$ (instead of equation (A20)) and equation (A21) of Lemma 3 becomes

$$
\begin{aligned}
& l_{p}[k-h+1]-l_{q}[k-h+1] \geq l_{p}\left[k-h-h_{q}^{i}[k-h]\right]- \\
& l_{q}\left[k-h-h_{q}^{i}[k-h]\right]-\sigma[k-h-\bar{h}], \\
& \text { entailing that condition (A22) becomes }
\end{aligned}
$$

$l_{p}[k+1-h]-l_{q}[k+1-h]>$

$>\varepsilon[k-h]-\sigma[k-h-\bar{h}] \geq 0$,

which holds by assumption A2.3. 


\section{APPENDIX 2}

If $x_{e}[k+1]>x_{e}[k]$, the upper plot of Fig. 4 shows that the quantity $\left(x_{e}[k+1]-x_{e}[k]\right) l_{e}\left(x_{e}[k+1]\right)$, equal to the area of the rectangle with bold lines, is larger than the integral $\int_{x_{e}[k]}^{x_{e}[k+1]} l_{e}(\xi) d \xi$, equal to the grey area. If $x_{e}[k+1]<x_{e}[k]$, the lower plot shows that $\left(x_{e}[k]-x_{e}[k+1]\right) l_{e}\left(x_{e}[k+1]\right)$, equal to the area of the rectangle with bold lines, is smaller than the integral $\int_{x_{e}[k+1]}^{x_{e}[k]} l_{e}(\xi) d \xi$, equal to the grey area.
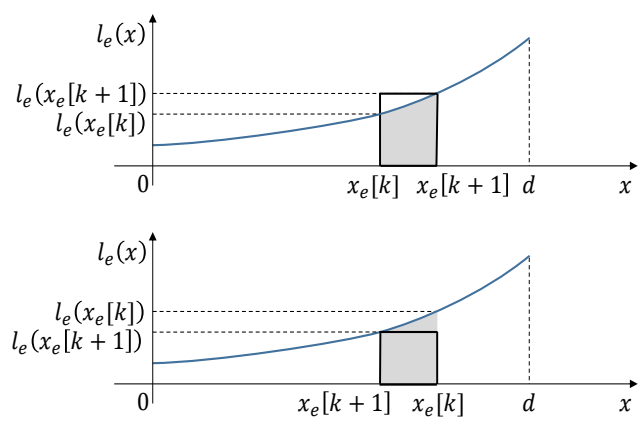

Figure 4. Geometrical considerations on the latency functions.

\section{REFERENCES}

[1] J. G. Wardrop, "Some Theoretical Aspects of Road Traffic Research," Proc. Inst. Civ. Eng., vol. 1, no. 3, pp. 325-362, May 1952.

[2] J. R. Correa and N. E. Stier-Moses, "Wardrop Equilibria," in Wiley Encyclopedia of Operations Research and Management Science, Hoboken, NJ, USA: John Wiley \& Sons, Inc., 2011.

[3] N. Nisan, T. Roughgarden, E. Tardos, and V. V. Vazirani, Eds., Algorithmic Game Theory. Cambridge: Cambridge University Press, 2007.

[4] A. Haurie and P. Marcotte, "On the relationship between Nash-Cournot and Wardrop equilibria," Networks, vol. 15, no. 3, pp. 295-308, 1985.

[5] R. L. Cruz and A. V. Santhanam, "Optimal routing, link scheduling and power control in multihop wireless networks," in IEEE INFOCOM 2003. Twenty-second Annual Joint Conference of the IEEE Computer and Communications Societies (IEEE Cat. No.03CH37428), vol. 1, pp. 702711.

[6] M. Geidl and G. Andersson, "Optimal Power Flow of Multiple Energy Carriers," IEEE Trans. Power Syst., vol. 22, no. 1, pp. 145-155, Feb. 2007.

[7] M. Papageorgiou, C. Kiakaki, V. Dinopoulou, A. Kotsialos, and Yibing Wang, "Review of road traffic control strategies," Proc. IEEE, vol. 91, no. 12, pp. 2043-2067, Dec. 2003.

[8] W. Wei, L. Wu, J. Wang, and S. Mei, "Network Equilibrium of Coupled Transportation and Power Distribution Systems," IEEE Trans. Smart Grid, vol. 9, no. 6, pp. 6764-6779, Nov. 2018.

[9] T. Roughgarden and É. Tardos, "How bad is selfish routing?," J. ACM,

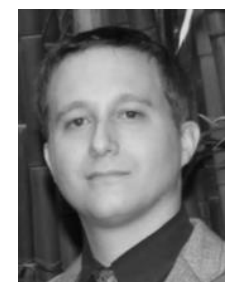

Alessandro Giuseppi received from the University of Rome "La Sapienza", Italy, his B.Sc. degree in Computer and Automation Engineering in 2014 and his M.Sc. degree in Control Engineering in 2016, both summa cum laude. Currently, he is a Ph.D. Candidate in Automatica at the Department of Computer, Control, and Management Engineering "Antonio Ruberti" of the same university. Since 2016, he has participated in 3 EU-funded projects. He authored about 15 journal and conference papers on the application of control theory to networked systems. vol. 49, no. 2, pp. 236-259, Mar. 2002.

[10] U. Bhaskar, K. Ligett, L. J. Schulman, and C. Swamy, "Achieving target equilibria in network routing games without knowing the latency functions," Games Econ. Behav., Mar. 2018.

[11] D. Barth, O. Bournez, O. Boussaton, and J. Cohen, "Distributed Learning of Wardrop Equilibria," in Unconventional Computing, Berlin, Heidelberg: Springer Berlin Heidelberg, 2008, pp. 19-32.

[12] H. Z. Aashtiani, H. Poorzahedy, and M. Nourinejad, "Extending Wardrop's First Principle for Capacitated Networks," Transp. A Transp. Sci., pp. 1-30, Jul. 2018.

[13] J. R. Correa, A. S. Schulz, and N. E. Stier-Moses, "Selfish Routing in Capacitated Networks," Math. Oper. Res., vol. 29, no. 4, pp. 961-976, Nov. 2004

[14] F. Ordóñez and N. E. Stier-Moses, "Wardrop Equilibria with RiskAverse Users," Transp. Sci., vol. 44, no. 1, pp. 63-86, Feb. 2010.

[15] F. Ordóñez and N. E. Stier-Moses, "Robust Wardrop Equilibrium," in Network Control and Optimization, Berlin, Heidelberg: Springer Berlin Heidelberg, 2007, pp. 247-256.

[16] A. Pietrabissa and V. Suraci, "Wardrop equilibrium on time-varying graphs," Automatica, vol. 84, pp. 159-165, Oct. 2017.

[17] M. Beckmann, C. B. McGuire, and C. B. Winsten, "Studies in the Economics of Transportation," 1956.

[18] V. S. Borkar and P. R. Kumar, "Dynamic Cesaro-Wardrop equilibration in networks," IEEE Trans. Automat. Contr., vol. 48, no. 3, pp. 382-396, Mar. 2003.

[19] S. Fischer, L. Olbrich, and B. Vöcking, "Approximating Wardrop equilibria with finitely many agents," Distrib. Comput., vol. 21, no. 2, pp. 129-139, Jul. 2008.

[20] S. Fischer and B. Vöcking, "Adaptive routing with stale information," Theor. Comput. Sci., vol. 410, no. 36, pp. 3357-3371, Aug. 2009.

[21] S. Fischer, H. Räcke, and B. Vöcking, "Fast Convergence to Wardrop Equilibria by Adaptive Sampling Methods," SIAM J. Comput., vol. 39, no. 8, pp. 3700-3735, 2010.

[22] G. Como, K. Savla, D. Acemoglu, M. A. Dahleh, and E. Frazzoli, "Robust Distributed Routing in Dynamical Networks-Part I: Locally Responsive Policies and Weak Resilience," IEEE Trans. Automat. Contr., vol. 58, no. 2, pp. 317-332, Feb. 2013.

[23] G. Como, K. Savla, D. Acemoglu, M. A. Dahleh, and E. Frazzoli, "Robust Distributed Routing in Dynamical Networks-Part II: Strong Resilience, Equilibrium Selection and Cascaded Failures," IEEE Trans. Automat. Contr., vol. 58, no. 2, pp. 333-348, Feb. 2013.

[24] A. Pietrabissa et al., "Lyapunov-Based Design of a Distributed Wardrop Load-Balancing Algorithm With Application to Software-Defined Networking," IEEE Trans. Control Syst. Technol., pp. 1-13, 2018.

[25] A. Pietrabissa and L. Ricciardi Celsi, "Discrete-Time Selfish Routing Converging to the Wardrop Equilibrium," IEEE Trans. Automat. Contr., vol. 64, no. 3, pp. 1288-1294, Mar. 2019.

[26] V. Sundarapandian, "An invariance principle for discrete-time nonlinear systems," Appl. Math. Lett., vol. 16, no. 1, pp. 85-91, 2003.

[27] W. Mei and F. Bullo, "LaSalle Invariance Principle for Discrete-time Dynamical Systems: A Concise and Self-contained Tutorial," arXiv Prepr. arXiv1710.03710, Oct. 2017.

[28] W. H. Sandholm, Population Games and Evolutionary Dynamics. the MIT Press, 2009.

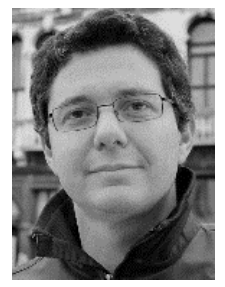

Antonio Pietrabissa is Associate Professor at the Department of Computer, Control, and Management Engineering "Antonio Ruberti" (DIAG) of the University of Rome "La Sapienza," where he received his degree in Electronics Engineering and his Ph.D. degree in Systems Engineering in 2000 and 2004,respectively, and where he teaches "Automatic Control" and "Process Automation". Since 2000, he has participated in about 20 EU and National research projects. His research focuses on the application of systems and control theory to the analysis and control of networks. $\mathrm{He}$ is author of more than 40 journal papers and 70 conference papers. 